\title{
Guidance of Block Needle Insertion by Electrical Nerve Stimulation: A Pilot Study of the Resulting Distribution of Injected Solution in Dogs
}

\author{
Marcel Rigaud, M.D. ${ }^{\star}$, Patrick Filip, M.D.†, Philipp Lirk, M.D. ${ }^{\prime}$, Andreas Fuchs, M.D. ${ }^{*}$ Geza \\ Gemes, M.D. , and Quinn Hogan, M.D.§ \\ ${ }^{*}$ Postdoctoral Fellow, Department of Anesthesiology, Medical College of Wisconsin, University Clinic for \\ Anesthesiology and Intensive Care Medicine, Medical University of Graz, Graz, Austria
}

$\uparrow$ Postdoctoral Fellow, Department of Anesthesiology, Medical College of Wisconsin, University Clinic for Anesthesiology and Intensive Care Medicine, Medical University of Graz, Graz, Austria

$\$$ Postdoctoral Fellow, Department of Anesthesiology and Critical Care Medicine, Medical University of Innsbruck, Innsbruck, Austria

$\S$ Professor, Department of Anesthesiology, Medical College of Wisconsin; Department of Anesthesiology, Zablocki VA Medical Center, Milwaukee, Wisconsin

\section{Abstract \\ Background-Little is known regarding the final needle tip location when various intensities of nerve stimulation are used to guide block needle insertion. Therefore, in control and hyperglycemic dogs, the authors examined whether lower-intensity stimulation results in injection closer to the sciatic nerve than higher-threshold stimulation.}

\begin{abstract}
Methods-During anesthesia, the sciatic nerve was approached with an insulated nerve block needle emitting either $1 \mathrm{~mA}$ (high-current group, $\mathrm{n}=9$ ) or $0.5 \mathrm{~mA}$ (low-current group, $\mathrm{n}=9$ in control $\operatorname{dogs}$ and $\mathrm{n}=6$ in hyperglycemic dogs). After positioning to obtain a distal motor response, the lowest current producing a response was identified, and ink $(0.5 \mathrm{ml})$ was injected. Frozen sections of the tissue revealed whether the ink was in contact with the epineurium of the nerve, distant to it, or within it.
\end{abstract}

\begin{abstract}
Results - In control dogs, the patterns of distribution using high-threshold (final current $0.99 \pm 0.03$ $\mathrm{mA}$, mean $\pm \mathrm{SD}$ ) and low-threshold (final current $0.33 \pm 0.08 \mathrm{~mA}$ ) stimulation equally showed ink that was in contact with the epineurium or distant to it. One needle placement in the high-threshold group resulted in intraneural injection. In hyperglycemic dogs, all needle insertions used a lowthreshold technique ( $\mathrm{n}=6$, final threshold $0.35 \pm 0.08 \mathrm{~mA})$, and all resulted in intraneural injections.

Conclusions-In normal dogs, current stimulation levels in the range of 0.33-1.0 mA result in needle placement comparably close to the sciatic nerve but do not correlate with distance from the target nerve. In this experimental design, low-threshold electrical stimulation does not offer satisfactory protection against intraneural injection in the presence of hyperglycemia.
\end{abstract}

A FUNDAMENTAL requirement during plexus or peripheral nerve blockade is deposition of the anesthetic solution close enough to the target nerves to achieve prompt and thorough interruption of nerve conduction. However, a final needle position must be achieved that also

Address correspondence to Dr. Hogan: Department of Anesthesiology, MEB, Department of Anesthesiology, 8701 Watertown Plank Road, Milwaukee, Wisconsin 53226. E-mail: qhogan@mcw.edu. 
avoids delivery of the anesthetic solution within the substance of the nerve, which may increase the risk of mechanical or toxic damage to the neurons. ${ }^{1,2}$ Various methods have been used to guide needle placement, including identification of neighboring bony and vascular landmarks and the generation of a sensory paresthesia by contact of the needle with the nerve. In addition, nerve imaging by ultrasound has recently become available. However, generation of a motor response by peripheral nerve stimulation (PNS) with current delivered through the injection needle remains a current standard technique for identifying the endpoint for needle advancement.

A recommended endpoint suitable for achieving successful block is the production of a specific distal motor event by a current less than $0.5 \mathrm{~mA},{ }^{3}$ with which high success rates are reported. ${ }^{4}$ However, important questions remain unanswered. Although there is anecdotal evidence that intraneural injection may produce intense pain, there are no clear data showing that intraneural injection always produces such an event, and clinical observations suggest that intraneural injection may not be painful. 5,6 That is, pain during injection may have high specificity as an indication of intraneural injection, but the sensitivity of this sign is unknown. The possibility that intraneural deposition of local anesthetic may in fact commonly accompany neural blockade guided by PNS has not been tested. It is believed that using nerve stimulation avoids the close needle contact and possible nerve injury that may occur using a paresthesia technique. ${ }^{7}$ For example, Choyce et al. ${ }^{8}$ have shown that the average current necessary to produce a motor stimulation is only $0.17 \mathrm{~mA}$ for axillary block needles that have been inserted to the point of producing a contact paresthesia. This indicates that using higher conventional thresholds for the PNS technique should result in stopping needle advancement at a greater distance. In contrast, however, Urmey and Stanton ${ }^{9}$ have shown that most needles positioned by paresthesia for interscalene block do not produce a motor response even with $1.0 \mathrm{~mA}$. Therefore, the relation between needle-nerve distance and motor stimulation current remains uncertain.

To better understand the needle placement that results during PNS-guided nerve blockade, we designed a study using a large mammal model and a tracer/histologic technique with a small injection volume for determining the depth of needle penetration with respect to the target nerve. It was not our intention with this experimental design to address distribution of clinically relevant volumes of injectate, but rather to mark the depth and track of needle placement. Further, we tested the hypothesis that the depth of needle placement is inversely related to threshold level. Finally, because there are reports that diabetes mellitus may decrease the responsiveness of peripheral nerves to electrical stimulation during nerve blockade, ${ }^{10,11}$ we investigated additional animals with experimental hyperglycemia.

\section{Materials and Methods}

\section{Dog Subjects}

The study was conducted in compliance with animal research regulations of the Medical College of Wisconsin and with the approval of the Institutional Animal Care and Use Committee (Milwaukee, Wisconsin). To limit the use of animal subjects while avoiding sequential survival surgeries in any one subject, mongrel dogs ( $\mathrm{n}=11$, both sexes, $25-35 \mathrm{~kg}$ ) were used from another study in a manner unlikely to affect the findings of either study. Specifically, injections were performed during the terminal, organ harvesting phase of openchest cardiovascular research protocols during general anesthesia $(200 \mathrm{mg} / \mathrm{kg}$ barbital sodium, $15 \mathrm{mg} / \mathrm{kg}$ pentobarbital) and mechanical ventilation. Coronary occlusion was followed by $3 \mathrm{~h}$ of reperfusion, during which animals showed no cardiogenic shock or failure, after which the nerve injection experiment was initiated. The cardiovascular study protocols variously included administration of intravenous sildenafil or simvastatin more than $3 \mathrm{~h}$ before the nerve injections. No local anesthetics were injected during the cardiovascular or nerve injection 
protocols. Four additional dogs were studied in which hyperglycemia had been induced by treatment with intravenous alloxan and streptozotocin. ${ }^{12}$ These animals developed sustained hyperglycemia (blood glucose $250-350 \mathrm{mg} / \mathrm{dl}$ ) without ketosis or renal insufficiency during the 3-week period between initiation of hyperglycemia and the terminal experiment.

\section{Sciatic Nerve Stimulation and Injection}

Hemodynamic conditions were stable throughout the experimental period. With the dog in a lateral position, a stimulating needle (22 gauge, 2-inch length, Stimuplex A needle; B. Braun, Bethlehem, PA) was inserted from the medial or lateral aspect at the mid-thigh level 1-2 cm posterior to femur. Stimulation through the needle was provided from a source (Digistim Plus 3 nerve stimulator; CCR Medical, Inc., St. Petersburg, FL) with the cathode lead on the needle and the anode lead clipped to muscles at the thoracic incision. This device produces a square wave monophasic pulse with a duration of $0.2 \mathrm{~ms}$, provides continuing readout of the stimulating current, and was tested on an oscilloscope for accuracy.

Animals were randomly assigned to one of two groups. In both, initial advancement of the needle was performed with 1-mA stimulation. When a distal motor response was achieved, typically extension at the ankle and/or plantar flexion of the toes, needle advancement was immediately halted, and its position was stabilized. The current was diminished until the motor response vanished and was then increased again to identify the minimal current sufficient to produce a motor response, which was tabulated as the "final threshold." In the high-current group, injection was performed at this point. In the low-current group, the current was set at $0.5 \mathrm{~mA}$, further advancement or needle redirection was performed until motor stimulation was achieved, and the final threshold was determined as described above and injection was performed. In all animals, $0.5 \mathrm{ml}$ of particulate black or orange ink was injected over $5 \mathrm{~s}$ at the final needle location, with $0.1 \mathrm{ml}$ of this total being injected during needle withdrawal to mark the needle track. This small volume was used because our goal was to accurately record the needle tip location and to identify whether solution injected through the needle at this location would enter the nerve. Therefore, the ink left behind served as a proxy for the tissue location of the needle tip at the moment of injection. A larger volume, such as a dose proportionate to a clinical injection, would tend to obscure this detail and substantially distort the anatomy. In most animals, both sciatic nerves were injected.

\section{Tissue Harvest and Section}

Immediately after injection, the animals were killed by removal of the heart. The posterior thigh tissues were removed en masse with care not to distort the tissues. The resulting specimen was marked with sutures for orientation and stored in a $-80^{\circ} \mathrm{C}$ freezer. The material was cryosectioned without fixation in 15- $\mu \mathrm{m}$ increments, and photographs were taken of the block every $300 \mu \mathrm{m}$ using a digital camera (d70, micro 105 lens, Nikon, Melville, NY; and a 1:2 teleconverter, Tamron Inc., Commack, NY) mounted on a tripod, with a scale included in the image. Sections were performed in a plane perpendicular to the axis of the sciatic nerve.

\section{Image Analysis}

Photographic series were examined without knowledge of the stimulation setting or presence of hyperglycemia. A standard process was used in which the injection site was first identified. Features identifying the section representing the needle position included accumulation of ink as a pool rather that occupying interstices in the tissue, a tapering pattern of ink in adjacent images, bleeding, and the residue of a track through the adjacent muscle marked by ink. Because of displacement of adjacent structures during palpation for injection and shift of layers after harvest, the needle track did not reliably indicate the exact path to the nerve, but was considered a reliable indicator that the injection site was within the sample. We thereafter examined images both proximally and distally to identify the deepest penetration of ink 
contiguous with that at the injection site. By this approach, even when the tissue layers at the point of injection were hard to discern exactly, we could determine the closest proximity of the needle to the sciatic nerve or its distal divisions at the moment of injection, assuming that the ink was unable to cross tissue barriers because of its particulate nature. Because our imaging does not allow reliable recognition of the perineurial layer, we categorized ink location as within the epineurium of the nerve, in contact with the epineurium, or distant from the nerve. In this final case, we measured the distance between the nerve and the closest ink.

\section{Statistical Analysis}

Cross-tabulation and the Pearson chi-square were used for nonparametric analysis of ink distribution patterns (Statistica 7.0; StatSoft, Tulsa OK). Significance levels were set at $P<$ 0.05 . Stimulation thresholds are presented as mean $\pm \mathrm{SD}$, and the significance of differences between groups was determined by the Student $t$ test.

\section{Results}

Distal motor stimulation was achieved in all animals. The injection site could be identified in 24 of 30 specimens, thus assuring that we did not miss the critical portion of the nerve in the harvest. Data were not used from the remaining 6 specimens. The pattern of ink distribution was readily categorized from examination of the photographed sections (figs. 1-3).

In the normoglycemic animals, the actual measured stimulation thresholds were higher in the high-threshold group compared with the low-threshold group $(P<0.001$; table 1$)$. The distribution patterns of ink (table 1) were not different between low and high stimulation threshold groups $(P=0.462)$, with both groups showing a mix of injections distant from the nerve and in contact with the nerve. (We acknowledge that this study's sample size cannot determine statistical similarity regarding this parameter but can only confirm differences.) In addition, one intraneural injection occurred in the high-threshold group. For injections that were distant from the nerve, the average distances in the low-and high-threshold groups were not different.

The six needle insertions in the four hyperglycemic dogs were all performed using a lowthreshold technique. No insertions in the hyperglycemic dogs used a high-threshold technique. The average stimulation threshold in the hyperglycemic group was the same as in the lowthreshold normoglycemic group, but the ink distribution patterns were significantly different $(P<0.001)$, with only intraneural patterns occurring in the hyperglycemic animals.

\section{Discussion}

Although monitoring a motor response during passage of current through the nerve block needle is a standard technique for guiding placement of the needle, there is minimal understanding of the final relation between needle and nerve when using this method. Even whether the solution is deposited within or outside the nerve has not been established when using various stimulation parameters. In our study, a high threshold of $1 \mathrm{~mA}$ resulted in a mix of distribution patterns comparable to that which occurs using a much lower threshold level of $0.33 \mathrm{~mA}$. While many cases in each category showed contact of the small volume of injected solution $(0.5 \mathrm{ml})$ with at least part of the nerve, others showed no solution actually reaching the epineurium. Because in all cases the solution reached within $0.5 \mathrm{~mm}$ of the nerve, successful block would likely have been achieved in all injections regardless of final stimulation threshold, because the larger volumes of clinical injections would provide a source from which diffusion of local anesthetic would ultimately block conduction. These anatomical results suggest that threshold differences in this range are not clinically relevant, as has been reported in studies of efficacy in patients. $9,13,14$ 
Although our study is not powered adequately to prove the similarity of the ink tracer location after insertion with high and low stimulation threshold, the lack of any trend showing a difference makes it unlikely that a large effect would be found with a larger sample size. The counterintuitive result that a lower final threshold does not deliver solution closer to the nerve may be due to the inherent inhomogeneity of the electrical conditions immediately surrounding the nerve. Consistent with this hypothesis, we have noted that minute movements of the stimulating needle that are too small to penetrate into a new tissue layer nonetheless may have substantial effects on the ability to produce a distal motor event, which probably represents contact of the needle tip with tissues of different resistance such as fat, fibrous tissue, or vascular elements.

Although a larger number of subjects in this study may have produced a difference between groups, we note that there was substantial variability in both the high-and low-threshold distribution patterns. Our results may also have been different if a noninsulated needle had been used. However, conduction through the tip alone is thought to produce a more precise placement than is achieved with a noninsulated shaft. ${ }^{15}$ Because the epineurium provides a relatively large resistive barrier to stimulation, ${ }^{16}$ variations in the thickness of this layer between subjects or on different fascicles that might have been approached by the stimulating needle may also account for variability in results that overwhelms any influence of stimulation current levels in the two groups. Our technique did not identify the location of the needle tip per se but rather the location of a small amount of solution injected through it. We used this approach because this leaves a secure trace of the needle's location at the moment of stimulation, and the toxic effect of injected anesthetic is considered the main contributor to nerve damage rather than mechanical damage from needle penetration per se. ${ }^{1,2}$ Finally, use of a stimulus duration other than the $0.2 \mathrm{~ms}$ used here may have resulted in different depths of needle placement. However, block success rates are indistinguishable even when 0.1- and 0.3ms durations are compared. 17

Two previous studies have examined the relation of stimulation current level to nerve/needle distance in animal models. ${ }^{15,18}$ The needle location was determined in both these studies by dissection, making accurate determination of injection depth relative to the nerve uncertain. The reported findings indicate that the needle was within $3 \mathrm{~mm}$ for thresholds of $0.37-0.47$ $\mathrm{mA}$ in rabbits ${ }^{18}$ and within $2 \mathrm{~mm}$ for thresholds of $0.9-1.2 \mathrm{~mA}$ in cats. ${ }^{15}$ The small size of the animals and decreased thickness of their epineurium may account for the greater distances at these stimulation levels. Also, our model duplicated a clinical technique, whereas a micromanipulator was used in these other studies.

In a small group of injections in hyperglycemic animals, we found that use of a low stimulation threshold uniformly resulted in intraneural injection. Although constraints on the availability of animals did not permit examination of a high-threshold group in hyperglycemic dogs, it is not likely that improved safety would be guaranteed, considering the lack of difference we found in normal animals using high and low thresholds. Clinical reports have observed unexpectedly high stimulation thresholds in diabetic patients, ${ }^{10}$ even when ultrasound imaging shows contact of the needle with the nerve. ${ }^{11}$ These observations imply that low or normal thresholds could require intraneural needle placement to achieve motor stimulation, as we observed in hyperglycemic dogs. Conduction velocity in diabetics is slowed because of numerous factors. ${ }^{19}$ Because longitudinal conduction of action potentials represents sequential excitation of segments of neuronal membrane, factors that diminish conduction velocity would also depress excitation by an external source of depolarization such as a needle electrode. Reduced excitability of peripheral nerves by depolarizing current has been confirmed in diabetics by clinical electrophysiologic examination. 20 
Clinical diabetes mellitus consists of a variety of pathogenic processes that accrue over time. Although the duration of hyperglycemia in the model we used was relatively brief, it nonetheless resulted in decreased conduction velocity in the timeframe we tested, attributable to disrupted neuronal metabolism ${ }^{21}$ and reduced endoneurial blood flow. ${ }^{22,23}$ Particularly important aspects of diabetic neuropathy are directly attributable to the effect of hyperglycemia via increased activity of the sorbitol pathway of glucose metabolism, which is activated in sensory neurons within hours of exposure to hyperglycemia and produces excitability deficits and sensory neuron dysfunction within 1-4 weeks. ${ }^{24-26}$

Our findings in addition indicate that intraneural injection may ensue during blockade guided by needle stimulation even in normoglycemic animals using a high stimulation threshold. Furthermore, compared with our protocol using a pulse duration of $0.2 \mathrm{~ms}$, it is possible that a commonly used shorter pulse duration of $0.1 \mathrm{~ms}{ }^{13}$ would increase the incidence of intraneural injection, because a shortened pulse duration results in a decreased potency for motor stimulation. ${ }^{27}$ The threshold for motor stimulation with needles placed intraneurally in surgically exposed pig nerves averages $0.43 \mathrm{~mA}$ and may range up to $1.8 \mathrm{~mA}{ }^{28}$ Although it is accepted that awake human subjects may experience pain during intraneural injection, there are no data to indicate what fraction of intraneural injections produce such a response, and it is known that intraneural injections may occur without a sensory event. $5,6,29,30$ Together, our data and these observations suggest that partial intraneural injection may be a common feature of nerve blocks using needle stimulation. In diabetics, a decreased sensitivity to electrical stimulation combined with diminished sensory function and increased sensitivity to local anesthetic toxicity 31 may particularly elevate the risk and consequence of unrecognized intraneural injections during nerve blocks guided by needle stimulation.

Certain limitations of our study must be emphasized. Although there is no direct manner in which previous coronary occlusion and sildenafil or simvastatin might alter the responsiveness of nerves to stimulation, secure conclusions will require confirmation without these potentially confounding influences. More importantly, the small number of subjects, particularly in the hyperglycemic group, means that these results must be considered preliminary and serve primarily to generate hypotheses. A much larger study with animal subjects subjected solely to block experiments, and confirmation in a study of patients, would be necessary before a change in clinical practice could be recommended on these matters. Finally, application of our findings to a clinical setting may be limited by an inability of some stimulation systems in clinical use to accurately duplicate the stimulation intensities described here.

We conclude that under study conditions that resemble clinical peripheral nerve blockade, both high- and low-current stimulation levels may result in needle placement close to the target nerve. The PNS technique does not preclude intraneural injection, especially in subjects with hyperglycemia. It is possible that additional monitoring and/or imaging techniques may be necessary to avoid intraneural injection.

\section{Acknowledgments}

Supported by an Erwin Schroedinger Fellowship (project J2695; to Dr. Rigaud) from the Austrian Science Fund, Vienna, Austria; and grant No. NS-42150 (to Dr. Hogan) from the National Institutes of Health, Bethesda, Maryland.

The authors thank John Tessmer, B.S., and John Krolikowski, B.S. (Laboratory Technicians, Medical College of Wisconsin, Milwaukee, Wisconsin), for expert assistance.

\section{References}

1. Selander D, Brattsand R, Lundborg G, Nordborg C, Olsson Y. Local anesthetics: Importance of mode of application, concentration and adrenaline for the appearance of nerve lesions. An experimental study 
of axonal degeneration and barrier damage after intrafascicular injection or topical application of bupivacaine (Marcain). Acta Anaesthesiol Scand 1979;23:127-36. [PubMed: 442943]

2. Gentili F, Hudson AR, Hunter D, Kline DG. Nerve injection injury with local anesthetic agents: A light and electron microscopic, fluorescent microscopic, and horseradish peroxidase study. Neurosurgery 1980;6:263-72. [PubMed: 7383289]

3. De Andres J, Sala-Blanch X. Peripheral nerve stimulation in the practice of brachial plexus anesthesia: A review. Reg Anesth Pain Med 2001;26:478-83. [PubMed: 11561271]

4. Franco CD, Vieira ZE. 1,001 subclavian perivascular brachial plexus blocks: Success with a nerve stimulator. Reg Anesth Pain Med 2000;25:41-6. [PubMed: 10660239]

5. Bigeleisen PE. Nerve puncture and apparent intraneural injection during ultrasound-guided axillary block does not invariably result in neurologic injury. Anesthesiology 2006;105:779-83. [PubMed: 17006077]

6. Sala-Blanch X, Pomes J, Matute P, Valls-Sole J, Carrera A, Tomas X, Garcia-Diez AI. Intraneural injection during anterior approach for sciatic nerve block. Anesthesiology 2004;101:1027-30. [PubMed: 15448541]

7. Neal JM. How close is close enough? Defining the "paresthesia chad. Reg Anesth Pain Med 2001;26:97-9. [PubMed: 11251130]

8. Choyce A, Chan VW, Middleton WJ, Knight PR, Peng P, McCartney CJ. What is the relationship between paresthesia and nerve stimulation for axillary brachial plexus block? Reg Anesth Pain Med 2001;26:100-4. [PubMed: 11251131]

9. Urmey WF, Stanton J. Inability to consistently elicit a motor response following sensory paresthesia during interscalene block administration. Anesthesiology 2002;96:552-4. [PubMed: 11873027]

10. Szerb J, Persaud D. Long current impulses may be required for nerve stimulation in patients with ischemic pain. Can J Anaesth 2005;52:963-6. [PubMed: 16251563]

11. Sites BD, Gallagher J, Sparks M. Ultrasound-guided popliteal block demonstrates an atypical motor response to nerve stimulation in 2 patients with diabetes mellitus. Reg Anesth Pain Med 2003;28:479_ 82. [PubMed: 14556142]

12. Kersten JR, Toller WG, Gross ER, Pagel PS, Warltier DC. Diabetes abolishes ischemic preconditioning: Role of glucose, insulin, and osmolality. Am J Physiol Heart Circ Physiol 2000;278:H1218-24. [PubMed: 10749717]

13. Gurnaney H, Ganesh A, Cucchiaro G. The relationship between current intensity for nerve stimulation and success of peripheral nerve blocks performed in pediatric patients under general anesthesia. Anesth Analg 2007;105:1605-9. [PubMed: 18042857]

14. Franco CD, Domashevich V, Voronov G, Rafizad AB, Jelev TJ. The supra-clavicular block with a nerve stimulator: To decrease or not to decrease, that is the question. Anesth Analg 2004;98:116771. [PubMed: 15041619]

15. Ford DJ, Pither C, Raj PP. Comparison of insulated and uninsulated needles for locating peripheral nerves with a peripheral nerve stimulator. Anesth Analg 1984;63:925-8. [PubMed: 6486492]

16. Rashbass C, Rushton WA. The relation of structure to the spread of excitation in the frog's sciatic trunk. J Physiol 1949;110:110-35. [PubMed: 15410489]

17. Koscielniak-Nielsen ZJ, Rassmussen H, Jepsen K. Effect of impulse duration on patients' perception of electrical stimulation and block effectiveness during axillary block in unsedated ambulatory patients. Reg Anesth Pain Med 2001;26:428-33. [PubMed: 11561262]

18. Sung DH. Locating the target nerve and injectate spread in rabbit sciatic nerve block. Reg Anesth Pain Med 2004;29:194-200. [PubMed: 15138902]

19. Quasthoff S. The role of axonal ion conductances in diabetic neuropathy: A review. Muscle Nerve 1998;21:1246-55. [PubMed: 9736052]

20. Krishnan AV, Kiernan MC. Altered nerve excitability properties in established diabetic neuropathy. Brain 2005;128:1178-87. [PubMed: 15758031]

21. Stevens MJ, Lattimer SA, Feldman EL, Helton ED, Millington DS, Sima AA, Greene DA. AcetylL-carnitine deficiency as a cause of altered nerve myo-inositol content, Na, K-ATPase activity, and motor conduction velocity in the streptozotocin-diabetic rat. Metabolism 1996;45:865-72. [PubMed: 8692023] 
22. Cameron NE, Cotter MA, Low PA. Nerve blood flow in early experimental diabetes in rats: Relation to conduction deficits. Am J Physiol 1991;261:E1-8. [PubMed: 1858864]

23. Pop-Busui R, Sullivan KA, Van Huysen C, Bayer L, Cao X, Towns R, Stevens MJ. Depletion of taurine in experimental diabetic neuropathy: Implications for nerve metabolic, vascular, and functional deficits. Exp Neurol 2001;168:259-72. [PubMed: 11259114]

24. Vincent AM, McLean LL, Backus C, Feldman EL. Short-term hyperglycemia produces oxidative damage and apoptosis in neurons. FASEB J 2005;19:638-40. [PubMed: 15677696]

25. Obrosova IG, Van Huysen C, Fathallah L, Cao XC, Greene DA, Stevens MJ. An aldose reductase inhibitor reverses early diabetes-induced changes in peripheral nerve function, metabolism, and antioxidative defense. FASEB J 2002;16:123-5. [PubMed: 11709499]

26. Dobretsov M, Hastings SL, Romanovsky D, Stimers JR, Zhang JM. Mechanical hyperalgesia in rat models of systemic and local hyperglycemia. Brain Res 2003;960:174-83. [PubMed: 12505670]

27. Hadzic A, Vloka JD, Claudio RE, Hadzic N, Thys DM, Santos AC. Electrical nerve localization: Effects of cutaneous electrode placement and duration of the stimulus on motor response. Anesthesiology 2004;100:1526-30. [PubMed: 15166574]

28. Chan VW, Brull R, McCartney CJ, Xu D, Abbas S, Shannon P. An ultrasono-graphic and histological study of intraneural injection and electrical stimulation in pigs. Anesth Analg 2007;104:1281-4. [PubMed: 17456687]

29. Bonner SM, Pridie AK. Sciatic nerve palsy following uneventful sciatic nerve block. Anaesthesia 1997;52:1205-7. [PubMed: 9485977]

30. Shah S, Hadzic A, Vloka JD, Cafferty MS, Moucha CS, Santos AC. Neurologic complication after anterior sciatic nerve block. Anesth Analg 2005;100:1515-7. [PubMed: 15845717]

31. Kalichman MW, Calcutt NA. Local anesthetic-induced conduction block and nerve fiber injury in streptozotocin-diabetic rats. Anesthesiology 1992;77:941-7. [PubMed: 1443749] 

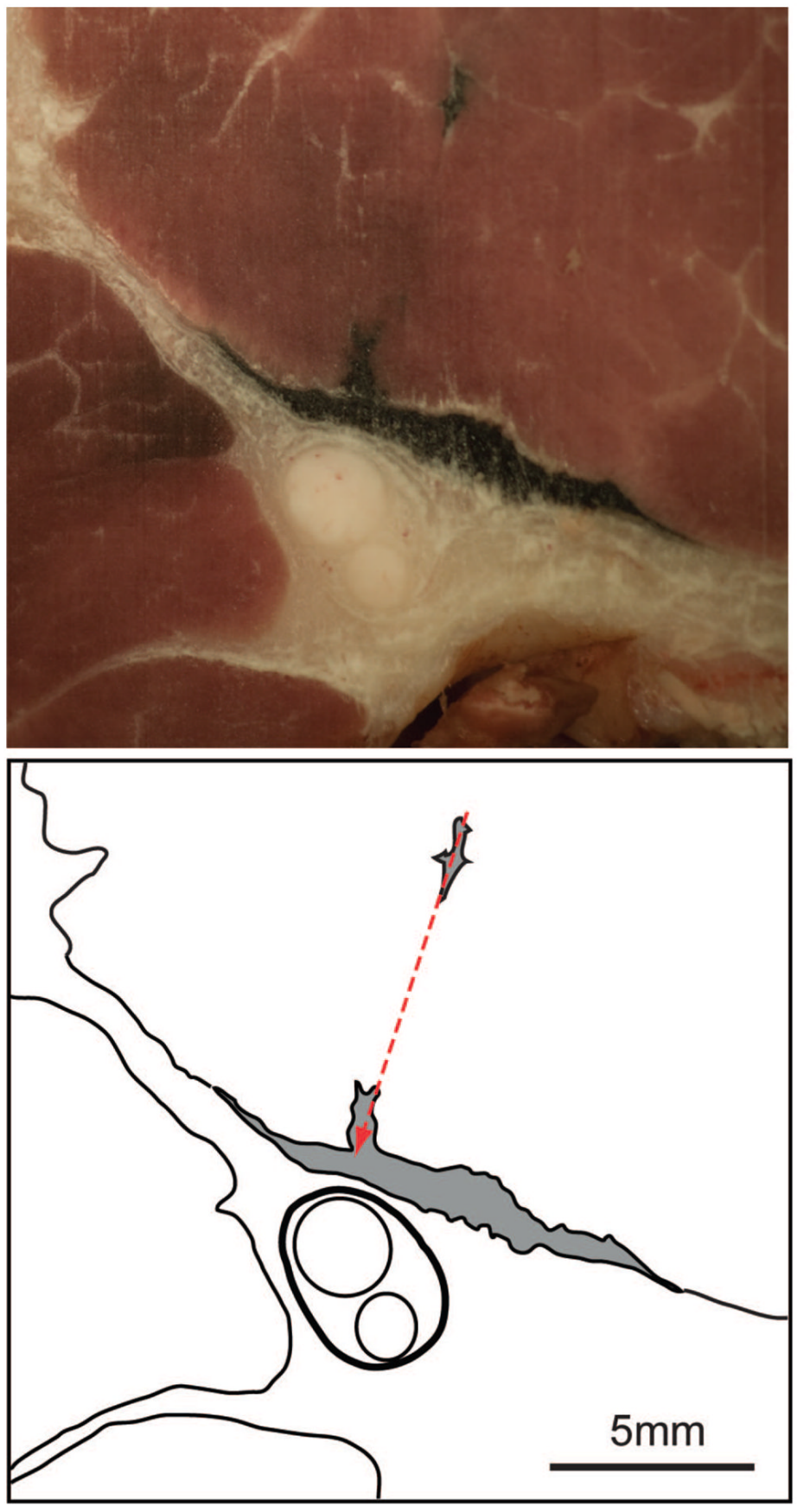

Fig. 1.

Photograph and reference tracing of a cryosection of the sciatic nerve and surrounding tissue after injection of $0.5 \mathrm{ml}$ black ink through a block needle that produced motor stimulation with a threshold of $1 \mathrm{~mA}$ in a control dog. The needle placement (arrow) is indicated by the residue of ink in the muscle from injection of an additional $0.1 \mathrm{ml}$ of ink as the needle was withdrawn. The epineurium of the sciatic nerve is shown in the tracing with a heavy line, and the main fascicles within the nerve are shown by lighter lines. The ink distribution is represented in gray shading. The closest proximity of ink to the nerve in this or other sections was $500 \mu \mathrm{m}$, and the injection pattern was categorized as distant from the nerve. 

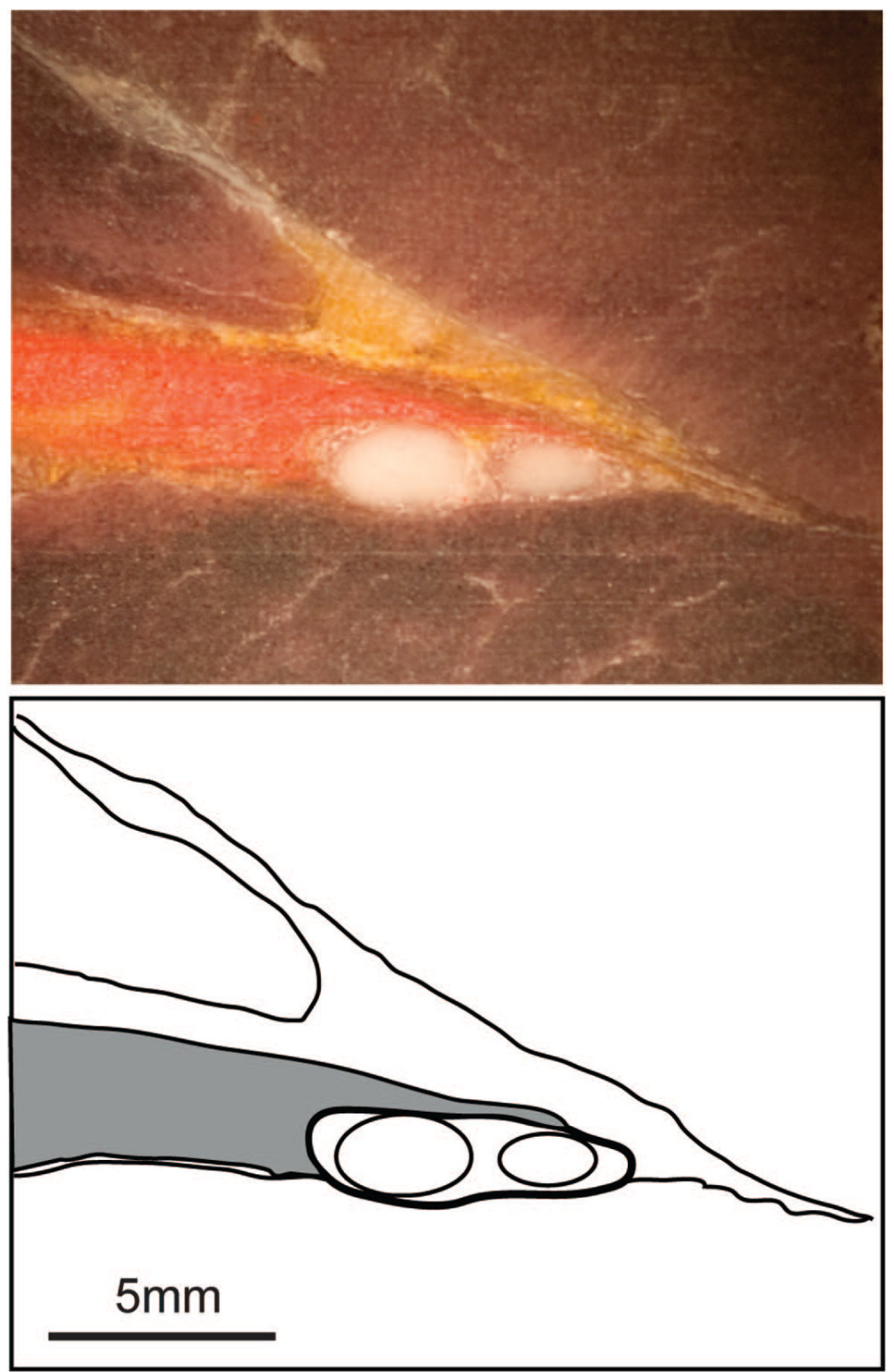

Fig. 2.

Photograph and reference tracing of a cryosection of the sciatic nerve and surrounding tissue after injection of $0.5 \mathrm{ml}$ orange ink through a block needle that produced motor stimulation with a threshold of $0.3 \mathrm{~mA}$ in a control dog. The needle track does not appear in this section. The epineurium of the sciatic nerve is shown in the tracing with a heavy line, and the main fascicles within the nerve are shown by lighter lines. The ink distribution is represented in gray shading. The ink layers against the external aspect of the epineurium of the nerve, and the injection pattern was categorized as in contact with the nerve. 
A
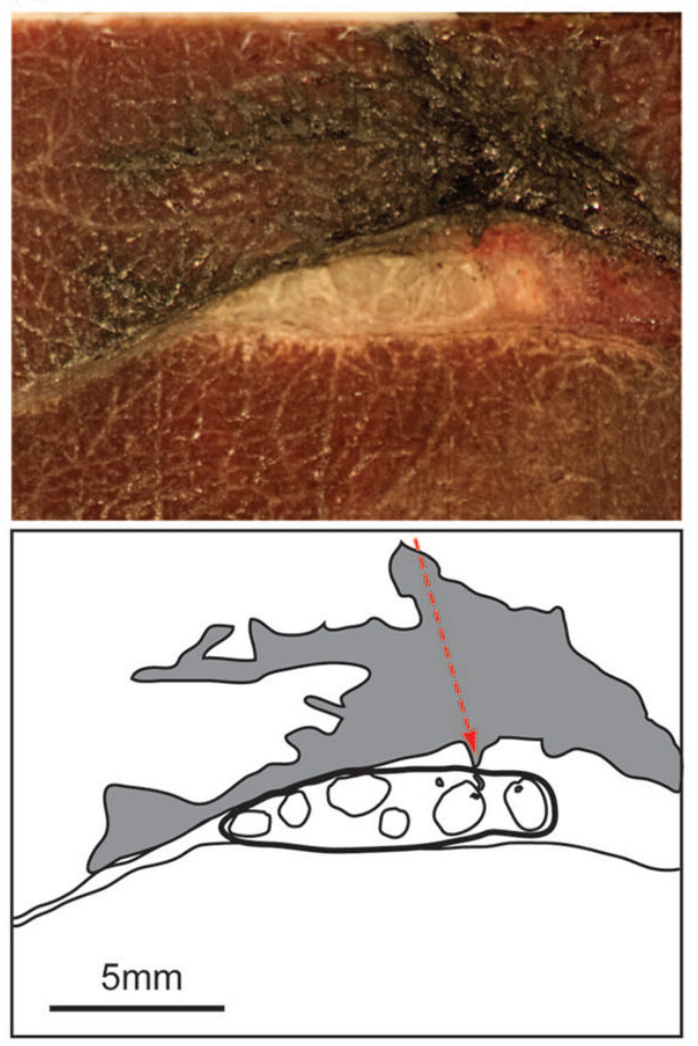

B
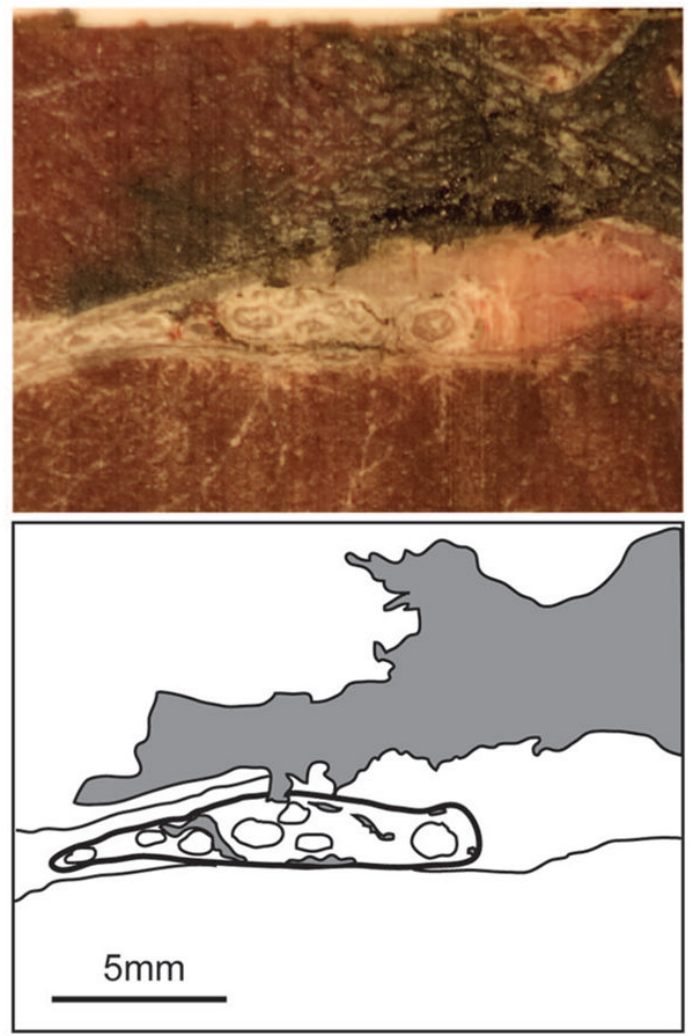

Fig. 3.

Photographs and reference tracings of cryosections of the sciatic nerve and surrounding tissue after injection of $0.5 \mathrm{ml}$ black ink through a block needle that produced motor stimulation with a threshold of $0.3 \mathrm{~mA}$ in a hyperglycemic $\operatorname{dog}$. $A$ is the likely site of needle placement (arrow) because there is an accumulation of free ink and there is bleeding in and adjacent to the nerve. A somewhat more distal image $(B)$ shows mores clearly that there are streaks of ink within the epineurium (heavy line) among the fascicles of the nerve (lighter lines). The ink distribution is represented in gray shading. The injection pattern was categorized as within the nerve. 
Rigaud et al.

Page 12

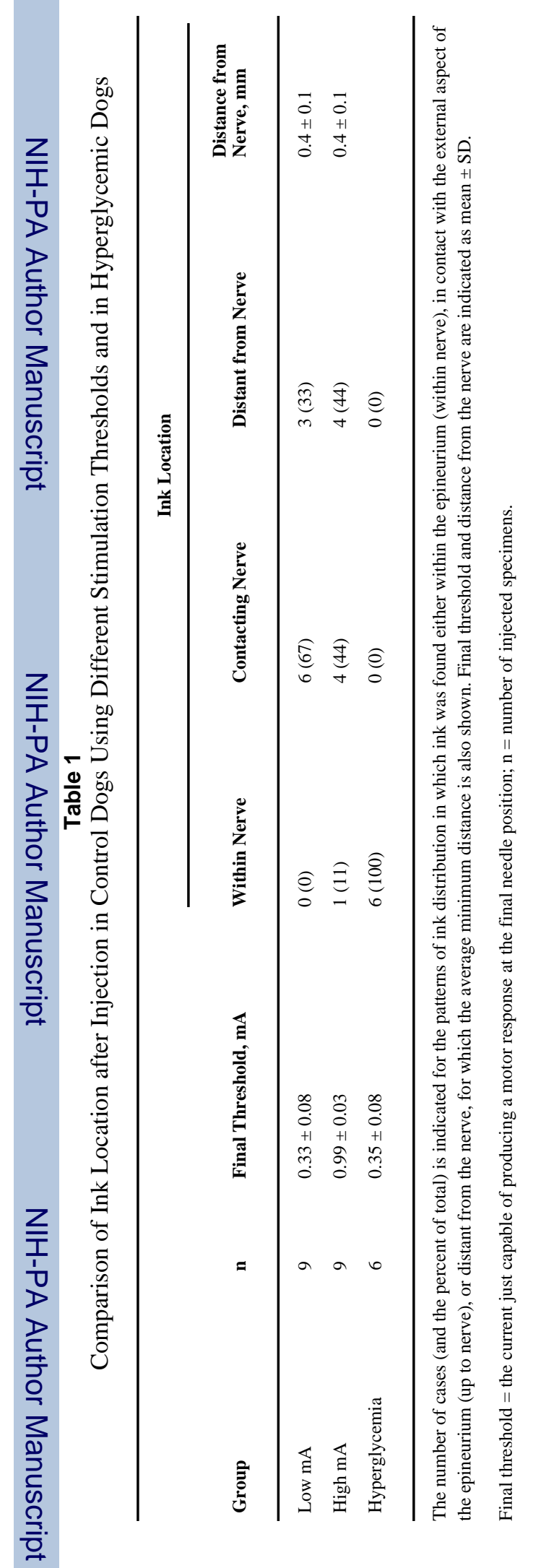

Anesthesiology. Author manuscript; available in PMC 2009 June 22. 